\title{
Frailties and copulas, not two of a kind
}

\author{
Klara Goethals ${ }^{\mathrm{a}}$, Paul Janssen ${ }^{\mathrm{b}}$ and Luc Duchateau ${ }^{\mathrm{a}, *}$ \\ ${ }^{a}$ Department of Comparative Physiology and Biometrics, Faculty of Veterinary Medicine, Ghent University, \\ Merelbeke, Belgium \\ ${ }^{\mathrm{b}}$ CenStat, Hasselt University, Hasselt, Belgium
}

\begin{abstract}
Bivariate survival data occur in diverse disciplines such as financial mathematics [The European Journal of Finance 15 (2009), 609-618] and biostatistics [Twin Res. 4 (2001), 407-411]. Different modeling approaches have been developed. Two standard approaches, the copula model and the frailty model, provide estimates of the correlation between event times in a cluster [The Frailty Model, Springer, New York, 2008].

A unified framework is proposed here for the copula model and the different types of frailty models, i.e., the univariate, shared and correlated frailty model, in order to evaluate similarities and differences between the models.

We further investigate the frailty effect at the event time scale; frailties operate at the hazard level, and therefore influence the event times in a nonlinear way, which leads in some instances to counterintuitive findings. For instance, in a cluster with frailty smaller than one, both event times will increase, but additionally also the difference between the two event times in the cluster will increase.
\end{abstract}

Keywords: Clayton copula, gamma frailty model, multivariate survival, shared frailty, correlated frailty, univariate frailty

\section{Introduction}

Different approaches have been proposed to model clustered survival data [3]. Frailty (conditional) and copula models not only accommodate for the correlation in the clustered data, but also provide estimates for the correlation, whereas marginal, fixed effects or stratified models only adjust parameter estimates for the correlation. In this paper, differences and similarities between the copula model and the different frailty models (the univariate, shared and correlated frailty model) are investigated for bivariate survival data, i.e., survival data with clusters of size 2 . We restrict our attention to Archimedean copulas having Laplace transforms as generators. For these copulas the resulting correlation structure is linked to the correlation structure of the most frequently used shared frailty models. For the sake of comparison, a unified framework for the different models is developed in Section 2. Using this framework, it will be shown that shared frailty models and copula models are different, although it has been claimed that these two models are equivalent if one uses the Laplace transform of the frailty density

\footnotetext{
*Address for correspondence: Luc Duchateau, Department of Comparative Physiology and Biometrics, Faculty of Veterinary Medicine, Ghent University, Salisburylaan 133, 9820 Merelbeke, Belgium. E-mail: luc.duchateau@ugent.be.
}

as generator of the Archimedean copula (see [6] for references). The claimed equivalence, however, is only true for the correlation structure between the marginal survival functions, but the marginal survival functions themselves are different in the two approaches. On the other hand, the univariate, shared and correlated frailty models are actually quite similar; they only differ in the way the frailties correlate.

The interpretation of the correlation and the heterogeneity parameter of the frailties is not straightforward, as the frailty operates on the hazard scale. For bivariate data, an interpretation of the heterogeneity induced by the frailties in terms of meaningful quantities, such as the median event time, has been presented previously [2]. It is however important to understand how frailties operate on event times. Investigating the effect of the frailty on the clustered event times is the topic of Section 3. A data example follows in Section 4 and conclusions are given in Section 5.

\section{A unifying modeling approach}

Copula models have been used most frequently for bivariate survival data, i.e., event times are clustered pairwise; we therefore focus our attention on such data, which can be presented by pairs $\left(Y_{i 1}, Y_{i 2}\right)$ where $i, i=$ 
$1, \ldots, s$, refers to the cluster and $Y_{i j}=\min \left(T_{i j}, C_{i j}\right)$ with $T_{i j}$ the event time and $C_{i j}$ the censoring time; $\delta_{i j}=1\left(T_{i j} \leqslant C_{i j}\right)$ denotes the censoring indicator. We further assume that the censoring time and the event time are statistically independent random variables and that the $T_{i 1}$ 's have the same distribution, $T_{i 1} \sim F_{1}$, as well as the $T_{i 2}$ 's, $T_{i 2} \sim F_{2}$.

Survival copula models represent the joint survival function $S_{p}\left(t_{1}, t_{2}\right)$ as a function of the marginal survival functions $S_{1, p}\left(t_{1}\right)$ and $S_{2, p}\left(t_{2}\right)$

$$
S_{p}\left(t_{1}, t_{2}\right)=C_{\vec{\theta}}\left(S_{1, p}\left(t_{1}\right), S_{2, p}\left(t_{2}\right)\right)
$$

with $C_{\vec{\theta}}$ the copula function with parameter vector $\vec{\theta}$ (for conditions on $C_{\vec{\theta}}$, see [8]).

The likelihood function can easily be obtained from the joint survival function

$$
\begin{aligned}
& \prod_{i=1}^{s}\left(f_{p}\left(y_{i 1}, y_{i 2}\right)\right)^{\delta_{i 1} \delta_{i 2}}\left(-\frac{\partial S_{p}\left(y_{i 1}, y_{i 2}\right)}{\partial y_{i 1}}\right)^{\delta_{i 1}\left(1-\delta_{i 2}\right)} \\
& \quad \times\left(-\frac{\partial S_{p}\left(y_{i 1}, y_{i 2}\right)}{\partial y_{i 2}}\right)^{\left(1-\delta_{i 1}\right) \delta_{i 2}} \\
& \quad \times\left(S_{p}\left(y_{i 1}, y_{i 2}\right)\right)^{\left(1-\delta_{i 1}\right)\left(1-\delta_{i 2}\right)}
\end{aligned}
$$

with $f_{p}\left(y_{i 1}, y_{i 2}\right)$ the joint density function.

We restrict our attention to Archimedean copulas, as they have a specific link with shared frailty models, as will be shown at the end of this section. The general expression for an Archimedean copula is given by

$$
S_{p}\left(t_{1}, t_{2}\right)=p\left[q\left(S_{1, p}\left(t_{1}\right)\right)+q\left(S_{2, p}\left(t_{2}\right)\right)\right]
$$

with $p(\cdot)$ a decreasing function defined on $[0, \infty]$ with values in $[0,1]$ and satisfying $p(0)=1$. Furthermore $p(\cdot)$ has positive second derivative and $q(\cdot)$ is the inverse function of $p(\cdot)$.

Taking, e.g., $p(v)=(1+\theta v)^{-1 / \theta}$ gives

$$
S_{p}\left(t_{1}, t_{2}\right)=\left(S_{1, p}^{-\theta}\left(t_{1}\right)+S_{2, p}^{-\theta}\left(t_{2}\right)-1\right)^{-1 / \theta} .
$$

The corresponding $C(v, w)=\left(v^{-\theta}+w^{-\theta}-1\right)^{-1 / \theta}$ is the Clayton copula [1].

We restrict to parametrical modeling for the marginal survival functions in this discussion; the corresponding parameters are collected in the vector $\vec{\xi}$. To link the copula models to the frailty models, we will consider the same shape parameter $\rho$ and scale parameters $\lambda_{1}=\lambda$ and $\lambda_{2}=\lambda \exp (\beta)$ leading to $\vec{\xi}^{t}=(\lambda, \rho, \beta)$.
The likelihood function (1) can be maximised simultaneously for $\theta$ and $\vec{\xi}$, but often the two-stage approach is used [4]: first estimate $\vec{\xi}$ by fitting the marginal models ([7] prove the consistency of these estimates); then insert these estimates in (1) and maximise with respect to $\theta$ (the only remaining parameter).

Frailty models (univariate, shared, correlated) are conditional (hazard) models. The frailty term in the hazard expression can be integrated out using the frailty density to obtain both the marginal and joint survival functions. The same type of expression (1) as for the likelihood function of the copula model can be used for the different frailty models; we only need to replace $S_{p}$, resp. $f_{p}$, with the appropriate joint survival function, resp. joint density.

The univariate, shared and correlated frailty models take form

$$
h_{i j}(t)=h_{0}(t) u_{i j} \exp \left(x_{i j} \beta\right),
$$

where, for simplicity, we think $x_{i 1}=0$ and $x_{i 2}=1$.

The only difference between the three models is the assumption put on the correlation between the frailties $U_{i 1}$ and $U_{i 2}$. In Section 4 different scenarios are given, each of them can be described adequately by one of the three models. For all three models, we have that

$$
\operatorname{Corr}\left(U_{i j}, U_{i^{\prime} j^{\prime}}\right)=0 \quad \text { with } i \neq i^{\prime} .
$$

For the univariate frailty model we assume that the $U_{i j}$ 's are i.i.d. and

$$
\operatorname{Corr}\left(U_{i 1}, U_{i 2}\right)=0 .
$$

For the shared frailty model we have $U_{i 1}=U_{i 2}=$ $U_{i}$; hence

$$
\operatorname{Corr}\left(U_{i 1}, U_{i 2}\right)=1,
$$

i.e., the frailty is the same for both subjects, the two subjects in a cluster 'share' the same frailty.

The correlated frailty model takes an intermediate position having a correlation $\rho_{c}$ that satisfies

$$
0 \leqslant \rho_{c}=\operatorname{Corr}\left(U_{i 1}, U_{i 2}\right) \leqslant 1 .
$$

The marginal survival functions take the same form for all the frailty models

$$
\begin{aligned}
S_{j, m}(t) & =\int_{0}^{\infty} \exp \left(-u H_{0}(t) \exp \left(x_{j} \beta\right)\right) f_{U}(u) \mathrm{d} u \\
& =L\left(H_{0}(t) \exp \left(x_{j} \beta\right)\right)
\end{aligned}
$$


with $L(\cdot)$ the Laplace transform of the frailty density function $f_{U}(\cdot)$.

A frequently used frailty density function is the one parameter gamma density function with mean one and variance $\theta$ with corresponding Laplace transform $L(s)=(1+\theta s)^{-1 / \theta}$ leading to marginal survival functions

$$
S_{j, m}(t)=\left(1+\theta H_{0}(t) \exp \left(x_{j} \beta\right)\right)^{-1 / \theta} .
$$

The joint survival function for the univariate frailty model, $S_{u}\left(t_{1}, t_{2}\right)$, is given by

$$
S_{u}\left(t_{1}, t_{2}\right)=S_{1, m}\left(t_{1}\right) S_{2, m}\left(t_{2}\right) \text {. }
$$

The joint survival function for the shared frailty model, $S_{s}\left(t_{1}, t_{2}\right)$, is given by

$$
\begin{aligned}
& S_{s}\left(t_{1}, t_{2}\right) \\
& =\int_{0}^{\infty} \exp \left(-u\left(H_{0}\left(t_{1}\right)\right.\right. \\
& \left.\left.\quad+H_{0}\left(t_{2}\right) \exp (\beta)\right)\right) f_{U}(u) \mathrm{d} u \\
& \quad \underset{\text { gamma }}{=}\left(1+\theta\left(H_{0}\left(t_{1}\right)+H_{0}\left(t_{2}\right) \exp (\beta)\right)\right. \\
&
\end{aligned}
$$

Using (4) $S_{s}\left(t_{1}, t_{2}\right)$ can be rewritten as

$$
S_{s}\left(t_{1}, t_{2}\right)=\left(S_{1, m}^{-\theta}\left(t_{1}\right)+S_{2, m}^{-\theta}\left(t_{2}\right)-1\right)^{-1 / \theta} .
$$

The shared gamma frailty model leads to a similar structure of the joint survival function (5) as that for the Clayton copula (2). This has often led to the conclusion that copula models are equivalent to shared frailty models given an adequate choice of the Laplace transform. This is, however, not true due to the fact that the marginal survival functions are different. Equivalence only exists in the special case of Weibull distributed event times combined with the positive stable Laplace transform [6].

To obtain the joint survival function for the correlated frailty model, the frailty structure must be specified further. We assume

$$
U_{i 1}=Z_{i 0}+Z_{i 1} \quad \text { and } \quad U_{i 2}=Z_{i 0}+Z_{i 2}
$$

with $Z_{i 0} \sim \operatorname{Gamma}\left(k_{0}, \lambda\right)$ and $Z_{i 1}, Z_{i 2} \sim \operatorname{Gamma}\left(k_{1}\right.$, $\lambda$ ). We further assume that $Z_{i 0}, Z_{i 1}$ and $Z_{i 2}$ are independent and that the vectors $\left(Z_{i 0}, Z_{i 1}, Z_{i 2}\right), i=$ $1, \ldots, s$, are independent.
From this it follows that $U_{i j} \sim \operatorname{Gamma}\left(k_{0}+k_{1}, \lambda\right)$. We set $k_{0}+k_{1}=\lambda$ so that

$$
\begin{aligned}
& \mathrm{E}\left(U_{i j}\right)=1, \quad \operatorname{Var}\left(U_{i j}\right)=\theta=1 / \lambda \quad \text { and } \\
& \operatorname{Corr}\left(U_{i 1}, U_{i 2}\right)=\rho_{c}=\frac{k_{0}}{k_{0}+k_{1}} .
\end{aligned}
$$

The joint survival function $S_{c}\left(t_{1}, t_{2}\right)$ then equals

$$
\begin{aligned}
& \int_{0}^{\infty} \int_{0}^{\infty} \int_{0}^{\infty} \exp \left(-\left(z_{0}+z_{1}\right) H_{0}\left(t_{1}\right)\right.\left.-\left(z_{0}+z_{2}\right) H_{0}\left(t_{2}\right) \exp (\beta)\right) \\
& \quad \times f_{Z_{0}}\left(z_{0}\right) f_{Z_{1}}\left(z_{1}\right) f_{Z_{2}}\left(z_{2}\right) \mathrm{d} z_{0} \mathrm{~d} z_{1} \mathrm{~d} z_{2} \\
&=\left(1+\theta H_{0}\left(t_{1}\right)\right)^{-\left(1-\rho_{c}\right) / \theta} \\
& \quad \times\left(1+\theta H_{0}\left(t_{2}\right) \exp (\beta)\right)^{-\left(1-\rho_{c}\right) / \theta} \\
& \times\left(1+\theta\left(H_{0}\left(t_{1}\right)+H_{0}\left(t_{2}\right) \exp (\beta)\right)\right)^{-\rho_{c} / \theta}
\end{aligned}
$$

which can be rewritten in terms of the marginal survival functions as

$$
\begin{aligned}
& S_{c}\left(t_{1}, t_{2}\right) \\
& \quad=\frac{\left(S_{1, m}\left(t_{1}\right)\right)^{1-\rho_{c}}\left(S_{2, m}\left(t_{2}\right)\right)^{1-\rho_{c}}}{\left(\left(S_{1, m}\left(t_{1}\right)\right)^{-\theta}+\left(S_{2, m}\left(t_{2}\right)\right)^{-\theta}-1\right)^{\rho_{c} / \theta}} .
\end{aligned}
$$

The corresponding likelihood is most often maximised through a two-stage approach. This means that the same parameter $\theta$ is estimated twice, once in the marginal survival functions, and once in the maximisation of the likelihood function (2). Yashin et al. [10] already note that, if two very different estimates for $\theta$ emerge from this two-stage approach, the conclusion should be that the proposed model does not fit the data well.

\section{How do frailties operate on event times?}

Frailties operate at the hazard level. This makes interpretation of the frailty effect on more relevant outcomes, such as the median event time, not straightforward. Duchateau and Janssen [2] demonstrated how the frailty effect can be converted to heterogeneity of meaningful outcomes such as the median event time.

The aim of this section is to demonstrate how frailties operate on individual event times making use of simulations. We generate bivariate survival data with 
conditional Weibull distributions, using the same scale parameter $\lambda$ and shape parameter $\rho$ for the two subjects in the same cluster (and $\beta$ can thus be dropped), and including (or not) a shared gamma frailty term with variance $\theta=0.5$. We randomly draw a number $z$ from the uniform distribution with support $[0,1]$. For a given $z$, the corresponding survival percentile, $t(z)$, is defined by the equation $\mathrm{P}(T>t(z))=z$ and thus

$$
t(z)=\left(\frac{-\log z}{\lambda}\right)^{1 / \rho}
$$

for the model without frailty term; and

$$
t(z \mid u)=\left(\frac{-\log z}{\lambda u}\right)^{1 / \rho}=t(z) u^{-1 / \rho}
$$

for the model with frailty.

The presence of the frailty makes the survival percentile a random variable that depends on the shape parameter $\rho$; $\rho$ values above (below) one lead to a decreasing (increasing) effect of the multiplicative frailty term (closer to 1 ). For instance, for $u=0.5$, the multiplicative frailty effect doubles for $\rho=0.5$ compared to the exponential distribution $(\rho=1)$ (Table 1).

In Fig. 1, univariate data are generated for the Weibull distribution with $\lambda=1$ and three different values for $\rho$ ( $\rho=0.83,1$ and 1.2) using the same randomly generated percentiles (appearing on the same line). The asterisks correspond to event times for the model without frailties, the diamonds to event times of the univariate frailty model, with the frailty term given in the right column of the picture. It is obvious that the inclusion of a frailty term increases the heterogeneity between the event times, with the largest effect observed for $\rho=0.83$.

In Fig. 2a, bivariate data are generated for the Weibull distribution with $\rho=1$, i.e., the exponential

Table 1

The effect of the frailty as a function of $\rho$ on the time to event

\begin{tabular}{llc}
\hline Frailty & $\rho$ & Multiplicative factor $u^{-1 / \rho}$ \\
\hline$u=2$ & 0.5 & 0.25 \\
& 1 & 0.50 \\
& 2 & 0.71 \\
$u=0.5$ & 0.5 & \\
& 1 & 4 \\
& 2 & 2 \\
& & 1.4 \\
\hline
\end{tabular}

Note: For a time to event equal to 1 without frailty, the last column also represents the equivalent time with frailty term. distribution. The asterisks correspond to event times for the model without frailties, the diamonds to event times of the shared frailty model, with the frailty term given in the right column of the picture. For clusters with frailty terms below (above) 1, the distance between the two event times increases (decreases) compared to the model without frailty terms. The cluster averages of the same data are given in Fig. 2b. It is clear that heterogeneity increases at the level of these averages when including frailty terms.

\section{Data example: Time to malaria}

We study time to malaria in children as a function of bednet use. Such bednets prevent bites of Anopheles mosquitos, the vector of the malaria parasites. In each of 150 villages, one child in one household is randomly assigned to receive a bednet, another child in another household is assigned to control (no bednet). Children are followed up for malaria for 400 days.

For such bivariate survival data with village as cluster, we can envisage the following scenarios:

(1) All children with the same treatment (bednet or not) have the same underlying hazard function.

(2) Children have varying hazard functions due to innate malaria susceptibility, unrelated to village.

(3) Villages have varying hazard functions due to Anopheles vector distribution, related to village.

(4) A combination of (2) and (3).

It is clear that scenario (1) leads to an ordinary survival model, scenario (2) to the univariate frailty model, scenario (3) to the shared frailty model and scenario (4) to the correlated frailty model.

The results of the different models are given in Table 2 .

According to the results in Table 2, a frailty term needs to be included as the heterogeneity parameter $\theta$ is large compared to its standard error for the shared and correlated frailty model. Amongst the frailty models, the shared frailty model is the preferred one, as the correlation can take any value for the correlation between the two frailties in a cluster, but converges here to the shared frailty model with correlation between the two frailties equal to 1 . Comparing the shared frailty model with the copula model is much more difficult as these models have different baseline hazards (see [3], p. 105). 


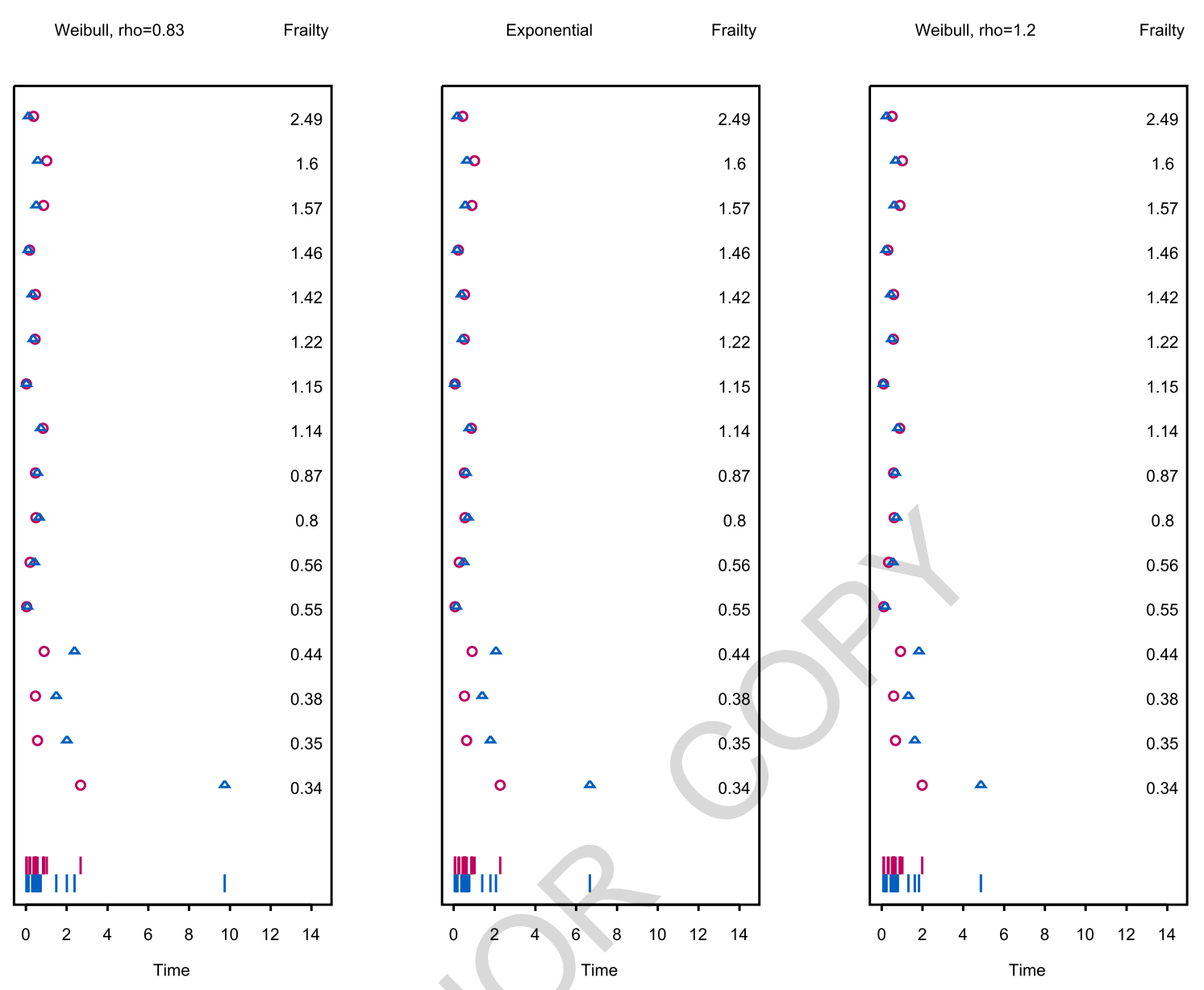

Fig. 1. Representation of univariate data simulated from a Weibull distribution with $\lambda=1$ and $\rho=0.83$ (left panel), $\rho=1$ (middle panel), $\rho=1.2$ (right panel). The circles correspond to the model without frailties, the triangles to the univariate frailty model (frailties are given in the right column of the figure). Each row corresponds to a randomly generated percentile. (Colors are visible in the online version of the article; http://dx.doi.org/10.3233/RDA-2012-0067.)

\section{Conclusions}

To analyse bivariate survival data, different models exist. In this paper, we studied frailty and copula models, as they both provide an estimate for the correlation in the data.

It has been shown, using the general framework, that the univariate and shared frailty model are special cases of the correlated frailty model; it can therefore be seen from the results of the analysis which of the three models appears to be more appropriate, as demonstrated in our example. As the correlated frailty model converges to the shared frailty model, the shared frailty model is the preferred model for our data.

Although the correlation structure between the marginal survival functions in the shared gamma frailty model is the same as in the Clayton copula, both approaches lead to quite different results because the marginal survival functions do not match. The marginal survival functions of the shared frailty model contain the heterogeneity parameter. We therefore fit quite different models when starting conditionally or marginally from a Weibull distribution. Which model is most appropriate depends therefore on the data at hand. Diagnostics need to be developed to enable the data-analyst to make a choice between a copula or a frailty model.

\section{Acknowledgement}

This research was supported by the Interuniversity Attraction Poles research network P6/STADEC of the Belgian State (Federal Office for Scientific, Technical and Cultural Affairs). 

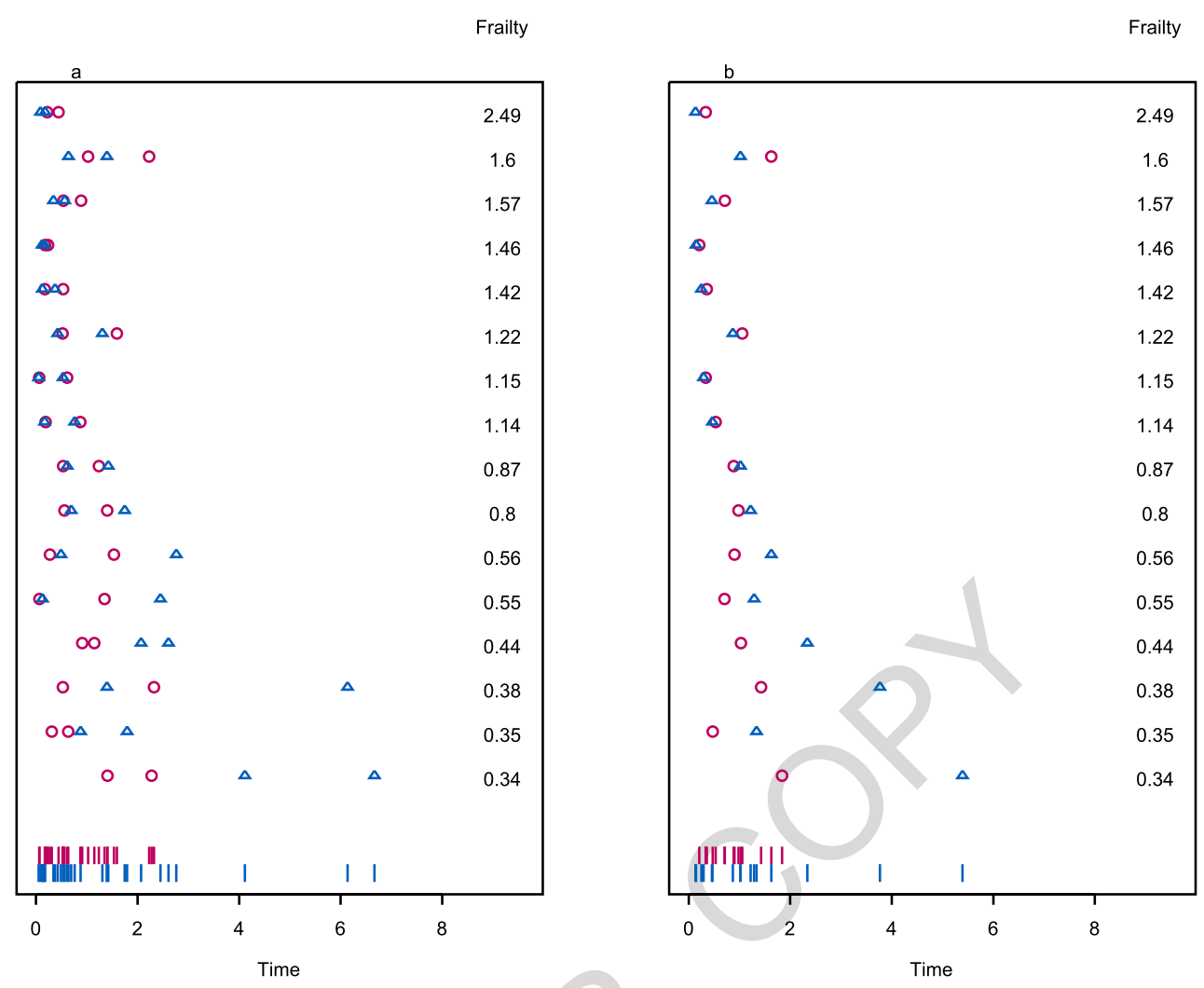

Fig. 2. Representation of bivariate data simulated from a Weibull distribution with $\lambda=1$ and $\rho=1$. The circles correspond to the model without frailties, the triangles to the shared frailty model (frailties are given in the right column of the figure). Each row corresponds to a randomly generated percentile. The left panel presents the two observations in a cluster, the right panel the mean of the cluster. (Colors are visible in the online version of the article; http://dx.doi.org/10.3233/RDA-2012-0067.)

Table 2

Results of fitting a parameter survival model with Weibull baseline hazard for the time to malaria data

\begin{tabular}{|c|c|c|c|c|c|}
\hline \multirow[t]{2}{*}{ Model } & \multicolumn{5}{|c|}{ Parameters } \\
\hline & $\lambda(\mathrm{se})$ & $\rho(\mathrm{se})$ & $\mathrm{HR}(\exp (\beta))(95 \% \mathrm{CI})$ & $\theta(\mathrm{se})$ & $\rho_{c}(\mathrm{se})$ \\
\hline \multirow[t]{2}{*}{ No frailty } & 0.016 & 0.752 & 0.81 & - & - \\
\hline & $(0.005)$ & $(0.045)$ & $(0.62-1.06)$ & & \\
\hline \multirow[t]{2}{*}{ Univariate } & 0.015 & 0.772 & 0.80 & 0.100 & - \\
\hline & $(0.006)$ & $(0.090)$ & $(0.60-1.07)$ & $(0.347)$ & \\
\hline \multirow[t]{2}{*}{ Shared } & 0.012 & 0.864 & 0.76 & 0.544 & - \\
\hline & $(0.004)$ & $(0.061)$ & $(0.58-1.02)$ & $(0.177)$ & \\
\hline \multirow[t]{2}{*}{ Correlated } & 0.012 & 0.864 & 0.76 & 0.544 & 1.000 \\
\hline & $(0.004)$ & $(0.061)$ & $(0.58-1.02)$ & $(0.177)$ & $(0.000)$ \\
\hline \multirow[t]{2}{*}{ Copula } & 0.015 & 0.800 & 0.61 & 1.024 & - \\
\hline & $(0.002)$ & $(0.049)$ & $(0.50-0.76)$ & $(0.259)$ & \\
\hline
\end{tabular}

Notes: The first model presents an ordinary survival analysis without taking the clustering in consideration. Next, the results are given for the univariate, shared and correlated frailty model with gamma frailty density. The last model is the Clayton copula model. 


\section{References}

[1] D.G. Clayton, A model for association in bivariate life tables and its application in epidemiological studies of family tendency in chronic disease incidence, Biometrika 65 (1978), 141-151.

[2] L. Duchateau and P. Janssen, Understanding heterogeneity in generalized mixed and frailty models, Amer. Statist. 59 (2005), 143-146.

[3] L. Duchateau and P. Janssen, The Frailty Model, Springer, New York, 2008.

[4] V. Durrleman, A. Nikeghbali and T. Roncalli, Which copula is the right one?, available at: http://ssrn.com/abstract=1032545, 2000.

[5] C. Genest, M. Gendron and M. Bourdeau-Brien, The advent of copulas in finance, The European Journal of Finance 15 (2009), 609-618.
[6] K. Goethals, P. Janssen and L. Duchateau, Frailty models and copulas: similarities and differences, J. Appl. Statist. 35 (2008), 1071-1079.

[7] W.J. Huster, R. Brookmeyer and S.G. Self, Modelling paired survival data with covariates, Biometrics 45 (1989), 145-156.

[8] R.B. Nelsen, An Introduction to Copulas, 2nd edn, Springer, New York, 2006.

[9] A. Wienke, N.V. Holm, A. Skytthe and A.I. Yashin, The heritability of mortality due to heart diseases: a correlated frailty model applied to Danish twins, Twin Res. 4 (2001), 407-411.

[10] A.I. Yashin, J.W. Vaupel and I.A. Iachine, Correlated individual frailty: an advantageous approach to survival analysis of bivariate data, Math. Popul. Stud. 5 (1995), 145-159. 